\title{
A Note on the Double $k$-class Estimator in Simultaneous Equations
}

\author{
Chuanming Gao, Kajal Lahiri* \\ Department of Economics, State University of New York at Albany, \\ Albany, NY 12222
}

\section{ABSTRACT}

Dwivedi and Srivastava (1984, DS) studied the exact finite sample properties of Nagar's (1962) double $k$-class estimator as continuous functions of its two characterizing scalars $k_{1}$ and $k_{2}$, and provided guidelines for their choice in empirical work. In this note we show that the empirical guidelines provided by DS are not entirely valid since they did not explore the complete range of the relevant parameter space in their numerical evaluations. We find that the optimal values of $k_{2}$ leading to unbiased and mean squared error (MSE) minimizing double $k$-class estimators are not symmetric with respect to the sign of the product $\rho \omega_{12}$, where $\rho$ is the correlation coefficient between the structural and reduced form errors, and $w_{12}$ is the covariance between the unrestricted reduced form errors. Specifically, when $\rho \omega_{12}$ is positive, the optimal value of $k_{2}$ is generally positive and greater than $k_{1}$, which partly explains the superior performance of Zellner's (1998) Bayesian Method of Moments (BMOM) and Extended MELO estimators reported in Tsurumi (1990).

\section{JEL classification: C30}

Keywords: Limited Information, Simultaneous Equations, Finite Sample, Mean Squared Error.

\footnotetext{
*Corresponding author. Tel: (518) 442 4758; fax: (518) 442 4736; e-mail: KLahiri@albany.edu.

We thank Terrence Kinal and two anonymous referees for helpful comments. All remaining shortcomings are of course our responsibility.
} 


\section{Introduction}

In an important paper, Dwivedi and Srivastava (1984, hereafter DS) studied the exact finite sample properties of Nagar's (1962) double $k$-class estimator. After deriving the first two moments of the estimator as continuous functions of the two characterizing scalars $k_{1}$ and $k_{2}$, they found that $k_{2}$ can be chosen such that a double $k$-class estimator is unbiased for a given $k_{1}$. DS also analyzed a result originally derived by Srivastava, Agnihotri and Dwivedi (1980) that it is always possible to choose $k_{2}$ such that a double $k$-class estimator has smaller mean squared error (MSE) than that of a $k$-class estimator. Through some numerical evaluations, DS provided guidelines on the choices of $k_{1}$ and $k_{2}$ for empirical work. They found that the value of $k_{2}$ which characterizes an unbiased double $k$-class estimator for a given $k_{1}$ between -1 and 1 is smaller than the value of $k_{1}$ and declines as the prespecified value of $k_{1}$ increases. Most of the time, this value of $k_{2}$ was found to be negative. They also concluded that the MSE minimizing value of $k^{*}$ is negative in majority of the cases and is larger in absolute value than the associated value of $k_{1} .{ }^{1}$

The main purpose of this note is to show that the empirical guidelines provided by DS are not entirely valid since they did not explore the whole range of the relevant parameter space in their numerical evaluations. DS adopted their model specifications from Sawa (1972) that were originally used to study the properties of single $k$-class estimators. We find that the optimal values of $k_{2}$ leading to unbiased and MSE minimizing double $k$-class estimators are not symmetric with respect to the sign of the product $\rho \omega_{12}$,

\footnotetext{
${ }^{1}$ See also Srivastava (1990) for a comprehensive survey on the properties of double $k$-class estimators.
} 
where $\rho$ is the correlation between the disturbances of the structural and the reduced form equations, and $\omega_{12}$ is the covariance between the unrestricted reduced form errors. For the single $k$-class estimator this asymmetry is not an issue. In all the specifications considered by DS, the implied value of $\rho$ is -0.514 and $\rho \omega_{12}$ is always negative. In addition we also derive a simple expression for the optimal value of $k_{2}$ such that a double $k$-class estimator has the smallest MSE for a given $k_{1}$. We find that when $\rho \omega_{12}$ is positive, the optimal value of $k_{2}$ is generally positive and greater than $k_{1}$.

\section{Finite sample moments}

Using the notations in DS, let us consider the following structural model:

$$
y_{1}=\beta y_{2}+X_{1} \gamma+u,
$$

where $y_{1}$ and $y_{2}$ are $T \times 1$ vectors of observations on the included endogenous variables; $X_{1}$ is a $T \times l$ matrix of observations on $l(<\Lambda)$ exogenous variables; $u$ is a $T \times 1$ vector of disturbances with zero mean and finite variance of $\sigma^{2}$. It is assumed that the structural equation is identified and the reduced form of the system is written as

$$
\left(\begin{array}{ll}
y_{1} & y_{2}
\end{array}\right)=X_{1}\left(\begin{array}{ll}
\pi_{11} & \pi_{21}
\end{array}\right)+X_{2}\left(\begin{array}{ll}
\pi_{12} & \pi_{22}
\end{array}\right)+\left(\begin{array}{ll}
v_{1} & v_{2}
\end{array}\right),
$$

where $X=\left(\begin{array}{ll}X_{1} & X_{2}\end{array}\right)$ is a $T \times \Lambda$ matrix with full column rank of observations on $\Lambda$ exogenous variables in the system with $X_{1}^{\prime} X_{2}=0$. The rows of $\left(\begin{array}{ll}v_{1} & v_{2}\end{array}\right)$ are assumed to be independently and identically distributed as $\mathrm{N}(0, \Omega)$, where $\Omega$ is $p d s$ and $\Omega=\left(\begin{array}{ll}\omega_{11} & \omega_{12} \\ \omega_{21} & \omega_{22}\end{array}\right)$. 
A double $k$-class estimator for the structural coefficient $\beta$, with characterizing scalars $k_{1}$ and $k_{2}$, can be expressed as

$$
\widehat{\beta}_{D K C}=\widehat{\beta}_{K C}+\left(k_{1}-k_{2}\right)\left(y_{2}^{\prime} A y_{2}\right)^{-1} y_{2}^{\prime} P y_{1},
$$

where $\widehat{\beta}_{K C}$ is the $k$-class estimator of $\beta$ with characterizing scalar $k_{1}$,

$$
\begin{aligned}
& P=I-X\left(X^{\prime} X\right)^{-1} X^{\prime}=I-X_{1}\left(X_{1}^{\prime} X_{1}\right)^{-1} X_{1}^{\prime}-X_{2}\left(X_{2}^{\prime} X_{2}\right)^{-1} X_{2}^{\prime}, \text { and } \\
& A=\left(1-k_{1}\right)\left[I-X_{1}\left(X_{1}^{\prime} X_{1}\right)^{-1} X_{1}^{\prime}\right]+k_{1} X_{2}\left(X_{2}^{\prime} X_{2}\right)^{-1} X_{2}^{\prime} .
\end{aligned}
$$

We also define the following:

$m=\frac{1}{2}(T-l), n=\frac{1}{2}(T-\Lambda), \delta=\frac{1}{2 \omega_{22}} \pi_{22}^{\prime} X_{2}^{\prime} X_{2} \pi_{22}$, and for non-negative integers $a, b, c$, and $d$,

$$
\begin{gathered}
\phi(a ; b)=e^{-\delta} \sum_{j=0}^{\infty} \frac{\Gamma(m-n+j-a)}{\Gamma(m-n+j+b)} \cdot \frac{\delta^{j}}{j !} \\
\psi_{-1 \leq k_{1}<1}(a ; b)=e^{-\delta} \sum_{\alpha=0}^{\infty} \sum_{j=0}^{\infty}(d \alpha+1) k_{1}^{\alpha} \frac{\Gamma(m+j-a-1) \Gamma(n+\alpha+b)}{\Gamma(m+j+\alpha+c) \Gamma(n)} \cdot \frac{\delta^{j}}{j !} .
\end{gathered}
$$

DS obtained the exact analytical expressions for mean and MSE of the double $k$-class estimator of $\beta$. When $-1 \leq k_{1}<1$ and $2 m \geq 1$, the bias of the double $k$-class estimator of $\beta$ is given by

$$
E\left(\widehat{\beta}_{D K C}\right)-\beta=\left(\beta-\frac{\omega_{12}}{\omega_{22}}\right)\left(\delta \psi_{0}(1 ; 0 ; 1)-1\right)+\left(k_{1}-k_{2}\right) \frac{\omega_{12}}{\omega_{22}} \psi_{0}(1 ; 1 ; 1) .
$$

When $-1 \leq k_{1}<1$ and $2 m \geq 3$, the MSE of the double $k$-class estimator of $\beta$ is given by

$$
\begin{aligned}
E\left(\widehat{\beta}_{D K C}-\beta\right)^{2}= & \left(\beta-\frac{\omega_{12}}{\omega_{22}}\right)^{2}+\left(k_{1}-k_{2}\right)^{2}\left(\frac{\omega_{12}}{\omega_{22}}\right)^{2} \psi_{1}(1 ; 2 ; 2) \\
& +\frac{\omega_{11.2}}{2 \omega_{22}}\left[\left(1-k_{2}\right)^{2} \psi_{1}(0 ; 1 ; 1)+(m-n) \psi_{1}(0 ; 0 ; 1)+\delta \psi_{1}(1 ; 0 ; 2)\right] \\
& +\delta\left(\beta-\frac{\omega_{12}}{\omega_{22}}\right)^{2}\left[\frac{1}{2} \psi_{1}(0 ; 0 ; 1)+\delta \psi_{1}(1 ; 0 ; 2)-2 \psi_{0}(1 ; 0 ; 1)\right] \\
& +2 \frac{\omega_{12}}{\omega_{22}}\left(\beta-\frac{\omega_{12}}{\omega_{22}}\right)\left(k_{1}-k_{2}\right)\left[\delta \psi_{1}(1 ; 1 ; 2)-\psi_{0}(1 ; 1 ; 1)\right],
\end{aligned}
$$


where $\omega_{11.2}=\omega_{11}-\omega_{12}^{2} / \omega_{22}$. The bias and MSE are similarly defined when $k_{1}$ is set at unity.

From (6) and (7), DS derived two interesting results. First, for a given $k_{1}$, the double $k$-class estimator is unbiased if the value of $k_{2}$ is set as

$$
\begin{aligned}
k_{u} & =k_{1}+\frac{\omega_{22}}{\omega_{12}}\left(\beta-\frac{\omega_{12}}{\omega_{22}}\right)\left[\frac{\delta \psi_{0}(1 ; 0 ; 1)-1}{\psi_{0}(1 ; 1 ; 1)}\right] \text { if }-1 \leq k_{1}<1, \\
& =1+\frac{\omega_{22}}{\omega_{12}}\left(\beta-\frac{\omega_{12}}{\omega_{22}}\right)\left[\frac{\delta \phi(0 ; 1)-1}{n \phi(1 ; 0)}\right] \text { if } k_{1}=1 .
\end{aligned}
$$

Second, the MSE of the double $k$-class estimator is less than that of $k$ class estimator with $-1 \leq k_{1}<1$ if the value of $k_{2}$ is between $k_{1}$ and $k^{*}$, with $k^{*}$ defined as

$$
\begin{aligned}
k^{*}= & k_{1}+\left\{\left[\left(1-k_{1}\right) \frac{\omega_{11.2}}{\omega_{22}} \psi_{1}(0 ; 1 ; 1)\right.\right. \\
& \left.+2 \frac{\omega_{12}}{\omega_{22}}\left(\beta-\frac{\omega_{12}}{\omega_{22}}\right)\left[\delta \psi_{1}(1 ; 1 ; 2)-\psi_{0}(1 ; 1 ; 1)\right]\right] \\
& \left./\left[\left(\frac{\omega_{12}}{\omega_{22}}\right)^{2} \psi_{1}(1 ; 2 ; 2)+\frac{\omega_{11.2}}{2 \omega_{22}} \psi_{1}(0 ; 1 ; 1)\right]\right\}
\end{aligned}
$$

If $k_{1}=1$,

$$
\begin{aligned}
k^{*}= & 1+\left\{\left[\frac{\omega_{12}}{\omega_{22}}\left(\beta-\frac{\omega_{12}}{\omega_{22}}\right)(\delta \phi(1 ; 1)-\phi(1 ; 0))\right]\right. \\
& \left./\left[\frac{\omega_{11.2}}{2 \omega_{22}} \phi(2 ; 0)+(n+1)\left(\frac{\omega_{12}}{\omega_{22}}\right)^{2} \phi(1 ; 0)\right]\right\} .
\end{aligned}
$$

Using (7), we further find that for a given $k_{1}$, the value of $k_{2}$ that results in the double $k$-class estimator with the minimum MSE is given by

$$
k^{* *}=\frac{1}{2}\left(k_{1}+k^{*}\right) .
$$

Even though (12) was not explicitly derived in DS, the above expression for the optimal $k_{2}$ is not surprising since the MSE of double $k$-class estimator is quadratic in $k_{2}$. 


\section{$3 \quad$ Numerical evaluations}

In order to get a feel about the magnitude and even the signs of $k_{u}$ and $k^{*}$, DS tabulated the values of $k_{u}$ and $k^{*}$ for selected values of $k_{1}$ and $\delta$ $\left(k_{1}=0, \pm 0.2, \pm 0.6, \pm 1\right.$ and $\left.\delta=1,10,25,50,100\right)$. Following Sawa (1972) and Srivastava et al.(1980), Dwivedi and Srivastava (1984) set

$$
\beta=1, \frac{\omega_{12}}{\omega_{22}}=0.4, \frac{\omega_{11.2}}{\omega_{22}}=1
$$

and considered the following three cases:

Case I. $\Lambda=5, l=2$, and $T=10,20,30,50$

Case II. $\Lambda=10, l=3$, and $T=20,30,50$

Case III. $\Lambda=15, l=5$, and $T=20,30,50$

For the sake of brevity, we only report results which corresponds to Case III in DS (i.e., $\Lambda=15, l=5$ ) with $T=50 .{ }^{2}$ Our Specification 1 has the same setup as in DS, and we try to duplicate the tabulated values of $k_{u}$ and $k^{*}$ in DS. In this specification, as in all the specifications considered by DS, the implied value of $\rho$ is -0.514 and $\rho \omega_{12} / \omega_{22}=-0.2056 .{ }^{3}$ Since $\omega_{22}>0$, the implied $\rho \omega_{12}$ is always negative in their setup. We then consider several alternative values of the parameters defined in (13) to demonstrate our point. In Specification 2 , we set $\beta=-1$, other parameters being the same. These parameter values imply $\rho=0.814$ and $\rho \omega_{12} / \omega_{22}=0.3256$. In Specification 3, we set $\omega_{12} / \omega_{22}=-0.4$, other things being the same as in Specification 1. This leads to an implied $\rho$ value of -0.814 and $\rho \omega_{12} / \omega_{22}=0.3256$. In Specification

\footnotetext{
${ }^{2}$ Numerical calculations are done using GAUSS for Windows Version 3.2.35 on a Pentium II $450 \mathrm{MHz}$ PC.

${ }^{3} \rho$ is derived using the following relationships: $\beta-\omega_{12} / \omega_{22}=-\rho \sqrt{\sigma^{2} / \omega_{22}}$, and $\sigma^{2} / \omega_{22}=\left(\omega_{11}-2 \beta \omega_{12}+\beta^{2} \omega_{22}\right) / \omega_{22}=\omega_{11.2} / \omega_{22}+\left(\beta-\omega_{12} / \omega_{22}\right)^{2}$.
} 
4, we further set $\omega_{12} / \omega_{22}=1.6$ yielding $\rho=0.514$ and $\rho \omega_{12} / \omega_{22}=0.8224$. The results from our numerical evaluations are collected in Tables 1, 2 and 3. We will first discuss results in Tables 1 and 2 .

Based on our extensive experiments with the specifications considered in DS, we found that there are substantial computational errors in the numerical evaluations reported by DS. In their Table 1 reporting values of $k_{u}$, only the results when $\delta=1$ and 10 have acceptable levels of accuracy. As $\delta$ gets larger (viz. $\delta=25,50,100$ ), their results became increasingly imprecise, and clearly unacceptable. This is seen if one compares $k_{u}$ values in their Table 1 (last panel of Case III, $m=22.5, n=17.5$ ) with those in our Table 1 (first panel). More strikingly, the values of $k^{*}$ reported in Table 2 of DS seem to be erroneous for all values of $\delta$ they considered. This is revealed by comparing the $k^{*}$ values in their Table 2 (last panel of Case III, $m=22.5, n=17.5$ ) with those in our Table 2 (first panel). For instance, DS reported that for our Specification 1, when $k_{1}=0, \delta=100$, the value of $k^{*}$ is -1071.807 , while it is -2.732 according to our calculation.

Overall we find that the value of $k^{*}$ is of the similar magnitude as $k_{u}$. It does not exhibit the tremendous variation as reported in DS. Also as a result of improved precision in our calculations, we find that the value of $k_{u}$ changes monotonically as $\delta$ increases for a given $-1<k_{1}<1$. Interestingly, when $k_{1}$ is set equal to 1 , we find that the value of $k_{u}$ is independent of $\delta .{ }^{4}$

Results from Specification 2 show that the optimal values of $k_{u}$ and $k^{*}$ may be all positive and greater than $k_{1}$, invalidating the major conclusion in DS

\footnotetext{
${ }^{4}$ We had difficulty in obtaining reliable results for specifications with $k_{1}=-1$ when $\delta=1$. Further investigation of these weak instrument situations is beyond the scope of this note. However, specifications with other values of $k_{1}$ demonstrate the inherent monotonic trend.
} 
that they take large negative values for most of the time and are less than $k_{2}$. These results may be easily explained by (6) and (7). First let us consider the determinants of the bias for the double $k$-class estimator. Observe that $\widehat{\beta}_{K C}$ is biased in the direction of $\rho$, as noted by Mariano (1982). Negative $\rho$ implies a downward bias, positive $\rho$ implies an upward bias in $\widehat{\beta}_{K C}$. Since for the single $k$-class estimator $k_{2}=k_{1}$, the first term $\left(\beta-\omega_{12} / \omega_{22}\right)\left(\delta \psi_{0}(1 ; 0 ; 1)-1\right)$ in (6) represents the bias of $\widehat{\beta}_{K C}$, and is of the same sign as that of $\rho$. In order to reduce the bias of the double $k$-class estimator, the second term $\left(k_{1}-k_{2}\right)\left(\omega_{12} / \omega_{22}\right) \psi_{0}(1 ; 1 ; 1)$ in $(6)$ should have sign opposite to that of $\rho$. Since $\psi_{0}(1 ; 1 ; 1)>0$, we require $\left(k_{1}-k_{2}\right) \omega_{12}$ to be of the opposite sign of $\rho$. Therefore when $\rho \omega_{12}<0$, i.e., $\rho$ and $\omega_{12}$ are of opposite signs, the value of $k_{u}$ should be less than $k_{1}$, as observed by DS. However when $\rho \omega_{12}>0$, the value of $k_{u}$ should be greater than $k_{1}$, as shown in our Specification 2 .

The dependence of the value of $k^{*}$ on the sign of $\rho \omega_{12}$ can be explained in a similar way. The first four terms in (7) do not depend on the sign of $\rho$ or $\omega_{12}$ explicitly. Regarding the last term in $(7)$, note that $\left[\delta \psi_{1}(1 ; 1 ; 2)-\right.$ $\left.\psi_{0}(1 ; 1 ; 1)\right]$ is negative and does not change sign for the wide range of values of $\delta$ considered. Thus, in order to make MSE of the double $k$-class estimator for a prespecified $k_{1}$ to be less than that of $\widehat{\beta}_{K C}$, we should choose the value of $k_{2}$ such that $\left(\omega_{12} / \omega_{22}\right)\left(\beta-\omega_{12} / \omega_{22}\right)\left(k_{1}-k_{2}\right)$ is negative. Therefore the value of $k^{*}$ should be selected in a similar way as for the value of $k_{u}$, depending on the sign of $\rho \omega_{12}$.

Note that the optimal values of $k_{u}$ (Table 1) and $k^{*}$ (Table 2) under Specification 3 are exactly the same as that under Specification 2. Also, in these two specifications, $\beta$ and $\omega_{12} / \omega_{22}$ values are set to be the same but of 
opposite signs, leaving $\rho \omega_{12} / \omega_{22}=0.3256$ in both cases. It implies that $k_{u}$ and $k^{*}$ are symmetric with respect to the signs of $\left(\omega_{12}, \rho\right)$. The results from Specification 4 show that both $k_{u}$ and $k^{*}$ move towards 1 as $k_{1}$ approaches 1. Again from Specifications 3 and 4 , we find that the optimal values of $k_{u}$ and $k^{*}$ are greater than $k_{1}$ when $\rho \omega_{12}>0$, but less than $k_{1}$ when $\rho \omega_{12}<0$.

Table 3 does not have a counterpart in DS and reports the optimal values of $k_{2}\left(k^{* *}\right)$ and the resulting mean squared errors (MSEs) of the double $k$-class estimator with characterizing scalars $k_{1}$ and $k^{* *}$. Comparing MSE figures under Specifications 2 and 3, we again find that they are symmetric with respect to the signs of $\left(\omega_{12}, \rho\right)$. It is shown that the values of $k^{* *}$ are much closer to $k_{u}$ than to $k^{*}$, suggesting that unbiasedness is the dominating criterion in fixing the MSE-minimizing value of $k_{2}$. When the value of $k_{1}$ is set at 1 , the value of $k^{* *}$ is very close or equal to 1 .

\section{Conclusions}

There has been a renewed interest in studying the properties of double $k$ class estimators. For instance, Zellner $(1986,1998)$ has shown that the extended minimum expected loss (ZEM) and the Bayesian Method of Moments (BMOM) estimators can be conveniently evaluated as double $k$-class estimators where the values of the two characterizing scalars $k_{1}$ and $k_{2}$ are uniquely determined by elegant balanced loss functions involving both 'goodness of fit' and 'precision of estimation' criteria. An attractive feature of BMOM approach is that it permits investigators to analyze given data when the form 
of the likelihood function is unknown. ${ }^{5}$ Tsurumi (1990) compared a number of Sampling theory and Bayesian estimators, and found that ZEM generally outperforms others, and is certainly among the top three in all cases.

Based on finite sample expressions for the first two moments of double $k$-class estimators, Dwivedi and Srivastava (1984) concluded that for a given value of $k_{1}$, the value of $k_{2}$ that yields unbiased and minimum MSE double $k$-class estimator is smaller than $k_{1}$, and is generally negative. We find that their guidelines on the choice of $k_{1}$ and $k_{2}$ are not entirely valid, and could be misleading. In their numerical evaluations they did not consider an important segment of the relevant parameter space. We point out that unlike the single $k$-class estimator, the properties of the double $k$-class estimator are severely affected by the signs of $\rho$ and $\omega_{12}$. The optimal value of $k_{2}$ for a given $k_{1}$ will be quite different depending on the sign of the product of $\rho$ and $\omega_{12}$. DS inadvertently considered only cases where $\rho \omega_{12}<0$, and found correctly that optimal values of $k_{2}$ should be less than the value of $k_{1}$. We, however, show that if $\rho \omega_{12}>0$, the optimal values of $k_{2}$ should be greater than $k_{1}$ and is generally positive. By comparing the optimal values of $k_{u}$ and $k^{* *}$, we also find that the unbiasedness criterion dominates in determining the optimal value of $k_{2}$.

\footnotetext{
${ }^{5}$ In a recent paper Zellner and Tobias (2001) extend previous BMOM results to show how information about a variance parameter and its relation to regression coefficients produces a rich class of postdata densities for regression parameters that maximizes entrophy.
} 


\section{References}

Dwivedi, T.D, and V.K. Srivastava (1984). Exact finite sample properties of double $k$-class estimators in simultaneous equations. Journal of Econometrics 25, 263-283.

Mariano, R.S. (1982). Analytical small-sample distribution theory in econometrics: the simultaneous-equation case. International Economic Review 23, 503-533.

Nagar, A.L. (1962). Double $k$-class estimators of parameters in simultaneous equations and their small sample properties. International Economic Review 3, 168-188.

Sawa, T. (1972). Finite-sample properties of the $k$-class estimators. Econometrica 40, 653-680.

Srivastava, V.K. (1990). Developments in double $k$-class estimators of parameters in structural equations. In: Carter, R.A.L., Dutta, J. and Ullah, A., eds., Contributions to Econometric Theory and Application, Essays in honour of A.L. Nagar (Springer-Verlag).

Srivastava, V.K., B.S. Agnihotri and T.D. Dwivedi (1980). Dominance of double $k$-class estimators in simultaneous equations. Annals of Institute of Statistical Mathematics 32, 387-392.

Srivastava, V.K., T.D. Dwivedi, M. Belinsky and R. Tiwari (1980). A numerical comparison of exact, large-sample and small-disturbance approximations of properties of $k$-class estimators. International Economic Review 21, 249-252.

Tsurumi, H. (1990). Comparing Bayesian and non-Bayesian limited information estimators. In: Geisser, S., Hodges, J.S., Press, S.J., Zellner, A., eds., Bayesian and Likelihood Methods in Statistics and Econometrics (North-Holland, Amsterdam).

Zellner, A. (1986). Further results on Bayesian minimum expected loss (MELO) estimates and posterior distributions for structural coefficients. In: Slottje, D., eds., Advances in Econometrics, Vol. 5, pp. 171-182.

Zellner, A. (1998). The finite sample properties of simultaneous equations' estimates and estimators: Bayesian and non-Bayesian approaches. Journal of Econometrics 83, 185-212. 
Zellner, A. and J. Tobias (2001). Further results on Bayesian Method of Moments analysis of the multiple regression model. International Economic Review 42, 121-140. 
Table 1. Values of $k_{2}\left(k_{\mathrm{u}}\right)$ leading to an unbiased double $k$-class estimator

\begin{tabular}{|c|c|c|c|c|c|}
\hline \multirow[b]{2}{*}{$k_{1}$} & \multicolumn{5}{|c|}{$\delta$} \\
\hline & 1 & 10 & 25 & 50 & 100 \\
\hline \multicolumn{6}{|c|}{ Specification 1. $\beta=1, \omega_{12} / \omega_{22}=0.4, \omega_{11.2} / \omega_{22}=1$} \\
\hline-1.0 & n.a. & -4.419 & -4.401 & -4.385 & -4.370 \\
\hline-0.8 & -3.935 & -3.917 & -3.898 & -3.882 & -3.868 \\
\hline-0.6 & -3.433 & -3.414 & -3.395 & -3.379 & -3.365 \\
\hline-0.2 & -2.429 & -2.407 & -2.388 & -2.373 & -2.361 \\
\hline 0.0 & -1.925 & -1.903 & -1.883 & -1.869 & -1.858 \\
\hline 0.2 & -1.420 & -1.397 & -1.378 & -1.365 & -1.356 \\
\hline 0.6 & -0.404 & -0.380 & -0.364 & -0.355 & -0.350 \\
\hline 0.8 & 0.112 & 0.134 & 0.145 & 0.150 & 0.154 \\
\hline 1.0 & 0.657 & 0.657 & 0.657 & 0.657 & 0.657 \\
\hline \multicolumn{6}{|c|}{ Specification $2 . \beta=-1, \omega_{12} / \omega_{22}=0.4, \omega_{11.2} / \omega_{22}=1$} \\
\hline-1.0 & n.a. & 6.979 & 6.936 & 6.898 & 6.862 \\
\hline-0.8 & 6.516 & 6.473 & 6.430 & 6.392 & 6.358 \\
\hline-0.6 & 6.011 & 5.966 & 5.923 & 5.885 & 5.853 \\
\hline-0.2 & 5.000 & 4.950 & 4.905 & 4.870 & 4.842 \\
\hline 0.0 & 4.492 & 4.439 & 4.395 & 4.361 & 4.336 \\
\hline 0.2 & 3.981 & 3.926 & 3.882 & 3.852 & 3.830 \\
\hline 0.6 & 2.944 & 2.886 & 2.850 & 2.829 & 2.816 \\
\hline 0.8 & 2.404 & 2.354 & 2.328 & 2.316 & 2.308 \\
\hline 1.0 & 1.800 & 1.800 & 1.800 & 1.800 & 1.800 \\
\hline \multicolumn{6}{|c|}{ Specification $3 . \beta=1, \omega_{12} / \omega_{22}=-0.4, \omega_{11.2} / \omega_{22}=1$} \\
\hline-1.0 & n.a. & 6.979 & 6.936 & 6.898 & 6.862 \\
\hline-0.8 & 6.516 & 6.473 & 6.430 & 6.392 & 6.358 \\
\hline-0.6 & 6.011 & 5.966 & 5.923 & 5.885 & 5.853 \\
\hline-0.2 & 5.000 & 4.950 & 4.905 & 4.870 & 4.842 \\
\hline 0.0 & 4.492 & 4.439 & 4.395 & 4.361 & 4.336 \\
\hline 0.2 & 3.981 & 3.926 & 3.882 & 3.852 & 3.830 \\
\hline 0.6 & 2.944 & 2.886 & 2.850 & 2.829 & 2.816 \\
\hline 0.8 & 2.404 & 2.354 & 2.328 & 2.316 & 2.308 \\
\hline 1.0 & 1.800 & 1.800 & 1.800 & 1.800 & 1.800 \\
\hline \multicolumn{6}{|c|}{ Specification $4 . \beta=1, \omega_{12} / \omega_{22}=1.6, \omega_{11.2} / \omega_{22}=1$} \\
\hline-1.0 & n.a. & -0.145 & -0.150 & -0.154 & -0.158 \\
\hline-0.8 & -0.016 & -0.021 & -0.025 & -0.029 & -0.033 \\
\hline-0.6 & 0.108 & 0.104 & 0.099 & 0.095 & 0.091 \\
\hline-0.2 & 0.357 & 0.352 & 0.347 & 0.343 & 0.340 \\
\hline 0.0 & 0.481 & 0.476 & 0.471 & 0.467 & 0.465 \\
\hline 0.2 & 0.605 & 0.599 & 0.595 & 0.591 & 0.589 \\
\hline 0.6 & 0.851 & 0.845 & 0.841 & 0.839 & 0.837 \\
\hline 0.8 & 0.972 & 0.966 & 0.964 & 0.962 & 0.962 \\
\hline 1.0 & 1.086 & 1.086 & 1.086 & 1.086 & 1.086 \\
\hline
\end{tabular}


Table 2. Values of $k^{*}$

\begin{tabular}{|c|c|c|c|c|c|}
\hline \multirow[b]{2}{*}{$k_{1}$} & \multicolumn{5}{|c|}{$\delta$} \\
\hline & 1 & 10 & 25 & 50 & 100 \\
\hline \multicolumn{6}{|c|}{ Specification 1. $\beta=1, \omega_{12} / \omega_{22}=0.4, \omega_{11.2} / \omega_{22}=1$} \\
\hline-1.0 & n.a. & -6.109 & -6.077 & -6.047 & -6.020 \\
\hline-0.8 & -5.494 & -5.459 & -5.424 & -5.392 & -5.364 \\
\hline-0.6 & -4.847 & -4.808 & -4.770 & -4.736 & -4.707 \\
\hline-0.2 & -3.549 & -3.501 & -3.456 & -3.420 & -3.392 \\
\hline 0.0 & -2.897 & -2.843 & -2.796 & -2.760 & -2.732 \\
\hline 0.2 & -2.241 & -2.181 & -2.132 & -2.097 & -2.072 \\
\hline 0.6 & -0.908 & -0.838 & -0.792 & -0.764 & -0.747 \\
\hline 0.8 & -0.214 & -0.147 & -0.110 & -0.093 & -0.083 \\
\hline 1.0 & 0.936 & 0.979 & 0.991 & 0.995 & 0.998 \\
\hline \multicolumn{6}{|c|}{ Specification 2. $\beta=-1, \omega_{12} / \omega_{22}=0.4, \omega_{11.2} / \omega_{22}=1$} \\
\hline-1.0 & n.a. & 12.983 & 12.876 & 12.778 & 12.687 \\
\hline-0.8 & 12.033 & 11.924 & 11.812 & 11.712 & 11.623 \\
\hline-0.6 & 10.979 & 10.862 & 10.746 & 10.645 & 10.557 \\
\hline-0.2 & 8.862 & 8.726 & 8.602 & 8.502 & 8.422 \\
\hline 0.0 & 7.796 & 7.650 & 7.523 & 7.426 & 7.353 \\
\hline 0.2 & 6.722 & 6.566 & 6.438 & 6.347 & 6.281 \\
\hline 0.6 & 4.527 & 4.355 & 4.240 & 4.173 & 4.131 \\
\hline 0.8 & 3.368 & 3.206 & 3.119 & 3.077 & 3.053 \\
\hline 1.0 & 1.149 & 1.049 & 1.022 & 1.011 & 1.006 \\
\hline \multicolumn{6}{|c|}{ Specification 3. $\beta=1, \omega_{12} / \omega_{22}=-0.4, \omega_{11.2} / \omega_{22}=1$} \\
\hline-1.0 & n.a. & 12.983 & 12.876 & 12.778 & 12.687 \\
\hline-0.8 & 12.033 & 11.924 & 11.812 & 11.712 & 11.623 \\
\hline-0.6 & 10.979 & 10.862 & 10.746 & 10.645 & 10.557 \\
\hline-0.2 & 8.862 & 8.726 & 8.602 & 8.502 & 8.422 \\
\hline 0.0 & 7.796 & 7.650 & 7.523 & 7.426 & 7.353 \\
\hline 0.2 & 6.722 & 6.566 & 6.438 & 6.347 & 6.281 \\
\hline 0.6 & 4.527 & 4.355 & 4.240 & 4.173 & 4.131 \\
\hline 0.8 & 3.368 & 3.206 & 3.119 & 3.077 & 3.053 \\
\hline 1.0 & 1.149 & 1.049 & 1.022 & 1.011 & 1.006 \\
\hline \multicolumn{6}{|c|}{ Specification 4. $\beta=1, \omega_{12} / \omega_{22}=1.6, \omega_{11.2} / \omega_{22}=1$} \\
\hline-1.0 & n.a. & 0.720 & 0.703 & 0.687 & 0.672 \\
\hline-0.8 & 0.782 & 0.765 & 0.747 & 0.732 & 0.718 \\
\hline-0.6 & 0.828 & 0.810 & 0.792 & 0.776 & 0.763 \\
\hline-0.2 & 0.917 & 0.897 & 0.879 & 0.865 & 0.853 \\
\hline 0.0 & 0.961 & 0.941 & 0.922 & 0.909 & 0.898 \\
\hline 0.2 & 1.005 & 0.983 & 0.965 & 0.952 & 0.943 \\
\hline 0.6 & 1.085 & 1.062 & 1.047 & 1.038 & 1.032 \\
\hline 0.8 & 1.117 & 1.096 & 1.085 & 1.079 & 1.076 \\
\hline 1.0 & 1.017 & 1.005 & 1.002 & 1.001 & 1.001 \\
\hline
\end{tabular}


Table 3. Values of $k_{2}\left(k^{* *}\right)$ with Minimum MSEs in parentheses

\begin{tabular}{|c|c|c|c|c|c|}
\hline \multirow[b]{2}{*}{$k_{1}$} & \multicolumn{5}{|c|}{$\delta$} \\
\hline & 1 & 10 & 25 & 50 & 100 \\
\hline \multicolumn{6}{|c|}{ Specification 1. $\beta=1, \omega_{12} / \omega_{22}=0.4, \omega_{11.2} / \omega_{22}=1$} \\
\hline-1.0 & n.a. & $-3.555(0.094)$ & $-3.538(0.057)$ & $-3.524(0.031)$ & $-3.510(0.015)$ \\
\hline-0.8 & $-3.147(0.1)$ & $-3.130(0$ & $12(0$. & $96(0$ & $82(0$ \\
\hline-0.6 & $-2.724(0.135)$ & $-2.704(0.086)$ & $-2.685(0.050)$ & $-2.668(0.026)$ & $-2.654(0.012)$ \\
\hline-0.2 & $-1.875(0.130)$ & $-1.850(0.077)$ & $-1.828(0.042)$ & $-1.810(0.021)$ & $-1.796(0.010)$ \\
\hline 0.0 & $-1.448(0.128)$ & $-1.421(0.072)$ & $-1.398(0.037)$ & $-1.380(0.019)$ & $-1.366(0.009)$ \\
\hline 0.2 & $-1.021(0.125)$ & $-0.991(0.066)$ & $-0.966(0.033)$ & $-0.949(0.017)$ & $-0.936(0.008)$ \\
\hline 0.6 & $-0.154(0.121)$ & $-0.119(0.054)$ & $-0.096(0.026)$ & $-0.082(0.014)$ & $-0.074(0.007)$ \\
\hline 0.8 & $0.293(0.127)$ & $0.327(0.051)$ & $0.345(0.024)$ & $0.354(0.013)$ & $0.359(0.007)$ \\
\hline 1.0 & $0.968(0.314)$ & $0.990(0.075)$ & $0.995(0.029)$ & $0.998(0.014)$ & $0.999(0.007)$ \\
\hline
\end{tabular}

Specification 2. $\beta=-1, \omega_{12} / \omega_{22}=0.4, \omega_{11.2} / \omega_{22}=1$

$\begin{array}{lcclll}-1.0 & \text { n.a. } & 5.992(0.119) & 5.938(0.073) & 5.889(0.042) & 5.844(0.021) \\ -0.8 & 5.617(0.176) & 5.562(0.117) & 5.506(0.071) & 5.456(0.040) & 5.411(0.020) \\ -0.6 & 5.190(0.179) & 5.131(0.115) & 5.073(0.068) & 5.022(0.037) & 4.979(0.018) \\ -0.2 & 4.331(0.187) & 4.263(0.111) & 4.201(0.062) & 4.151(0.033) & 4.111(0.017) \\ 0.0 & 3.898(0.194) & 3.825(0.109) & 3.761(0.059) & 3.713(0.032) & 3.676(0.016) \\ 0.2 & 3.461(0.204) & 3.383(0.108) & 3.319(0.056) & 3.273(0.030) & 3.240(0.015) \\ 0.6 & 2.563(0.253) & 2.477(0.110) & 2.420(0.053) & 2.387(0.028) & 2.366(0.014) \\ 0.8 & 2.084(0.325) & 2.003(0.119) & 1.960(0.055) & 1.938(0.029) & 1.926(0.015) \\ 1.0 & 1.075(1.249) & 1.024(0.238) & 1.011(0.080) & 1.006(0.035) & 1.003(0.016)\end{array}$

Specification 3. $\beta=1, \omega_{12} / \omega_{22}=-0.4, \omega_{11.2} / \omega_{22}=1$

$\begin{array}{lcclll}-1.0 & \text { n.a. } & 5.992(0.119) & 5.938(0.073) & 5.889(0.042) & 5.844(0.021) \\ -0.8 & 5.617(0.176) & 5.562(0.117) & 5.506(0.071) & 5.456(0.040) & 5.411(0.020) \\ -0.6 & 5.190(0.179) & 5.131(0.115) & 5.073(0.068) & 5.022(0.037) & 4.979(0.018) \\ -0.2 & 4.331(0.187) & 4.263(0.111) & 4.201(0.062) & 4.151(0.033) & 4.111(0.017) \\ 0.0 & 3.898(0.194) & 3.825(0.109) & 3.761(0.059) & 3.713(0.032) & 3.676(0.016) \\ 0.2 & 3.461(0.204) & 3.383(0.108) & 3.319(0.056) & 3.273(0.030) & 3.240(0.015) \\ 0.6 & 2.563(0.253) & 2.477(0.110) & 2.420(0.053) & 2.387(0.028) & 2.366(0.014) \\ 0.8 & 2.084(0.325) & 2.003(0.119) & 1.960(0.055) & 1.938(0.029) & 1.926(0.015) \\ 1.0 & 1.075(1.249) & 1.024(0.238) & 1.011(0.080) & 1.006(0.035) & 1.003(0.016)\end{array}$

Specification 4. $\beta=1, \omega_{12} / \omega_{22}=1.6, \omega_{11.2} / \omega_{22}=1$

\begin{tabular}{cccccc}
-1.0 & n.a. & $-0.140(0.009)$ & $-0.149(0.008)$ & $-0.157(0.006)$ & $-0.164(0.004)$ \\
-0.8 & $-0.009(0.011)$ & $-0.018(0.010)$ & $-0.026(0.008)$ & $-0.034(0.007)$ & $-0.041(0.004)$ \\
-0.6 & $0.114(0.011)$ & $0.105(0.010)$ & $0.096(0.009)$ & $0.088(0.007)$ & $0.081(0.005)$ \\
-0.2 & $0.359(0.013)$ & $0.349(0.012)$ & $0.340(0.010)$ & $0.332(0.007)$ & $0.327(0.005)$ \\
0.0 & $0.481(0.014)$ & $0.470(0.013)$ & $0.461(0.011)$ & $0.454(0.008)$ & $0.449(0.005)$ \\
0.2 & $0.602(0.017)$ & $0.591(0.015)$ & $0.582(0.012)$ & $0.576(0.009)$ & $0.571(0.005)$ \\
0.6 & $0.842(0.032)$ & $0.831(0.024)$ & $0.823(0.016)$ & $0.819(0.010)$ & $0.816(0.006)$ \\
0.8 & $0.959(0.057)$ & $0.948(0.034)$ & $0.942(0.020)$ & $0.940(0.011)$ & $0.938(0.006)$ \\
1.0 & $1.008(0.312)$ & $1.003(0.074)$ & $1.001(0.029)$ & $1.001(0.014)$ & $1.000(0.007)$ \\
\hline
\end{tabular}

